\title{
A sub-femtojoule electrical spin-switch based on optically trapped polariton condensates
}

Alexander Dreismann ${ }^{1 *}$, Hamid Ohadi ${ }^{1}$, Yago del Valle-Inclan Redondo ${ }^{1}$, Ryan Balili ${ }^{1}$, Yuri G. Rubo ${ }^{2}$, Simeon I. Tsintzos ${ }^{3}$, George Deligeorgis ${ }^{3}$, Zacharias Hatzopoulos ${ }^{3,4}$, Pavlos G. Savvidis ${ }^{1,3,5}$ and Jeremy J. Baumberg ${ }^{1+}$

Affiliations: ${ }^{1}$ Department of Physics, Cavendish Laboratory, University of Cambridge, Cambridge CB3 OHE, United Kingdom

${ }^{2}$ Instituto de Energías Renovables, Universidad Nacional Autónoma de México, Temixco, Morelos, 62580, Mexico

${ }^{3}$ Foundation for Research and Technology-Hellas, Institute of Electronic Structure and Laser, 71110 Heraklion, Crete, Greece

${ }^{4}$ CCQCN, Department of Physics, University of Crete, 71003 Heraklion, Crete, Greece

${ }^{5}$ Department of Materials Science and Technology, University of Crete, 71003 Heraklion, Crete, Greece

*ad635@cam.ac.uk, ${ }^{\dagger}$ iib12@cam.ac.uk

Practical challenges to extrapolating Moore's law favour alternatives to electrons as information carriers. Two promising candidates are spin-based and alloptical architectures, the former offering lower energy consumption $^{1}$, the latter superior signal transfer down to the level of chip-interconnects ${ }^{2}$. Polaritons - spinor quasi-particles composed of semiconductor excitons and microcavity photons - directly couple exciton spins and photon polarisations, combining the advantages of both approaches. However their implementation for spintronics has been hindered because polariton spins can only be manipulated optically ${ }^{3,4}$ or by strong magnetic fields $s^{5,6}$. Here we use an external electric field to directly control the spin of a polariton condensate, bias-tuning the emission polarisation. The nonlinear spin dynamics offers an alternative route to switching, allowing us to realise an electrical spin-switch exhibiting ultralow switching energies of order $0.5 \mathrm{fJ}$. Our results lay the foundation for development of devices based on the electro-optical control of coherent spin-ensembles on a chip.

Polaritons result from strong coupling of quantum-well (QW) excitons and photons in semiconductor microcavities. Their properties are determined by their constituents: from their photonic fraction comes a small effective mass $\left(\sim 10^{-4} m_{e}\right)$ and propagation over long distances at high speeds, while their excitonic component imparts strong self-interactions, resulting in large nonlinearities ${ }^{7,8}$. These characteristics favour Bosestimulated condensation into macroscopic quantum states, fully-coherent light-matter waves that spread over tens of microns and manifest a number of properties resembling atomic Bose-Einstein condensates and superfluids ${ }^{9-11}$. A number of polariton-based devices have been reported in the literature, including ultra-low power all-optical transistors and switches ${ }^{12,13}$.

Polaritons have two spin projections $s_{Z}= \pm 1$ arising from the coupling between spin-up (-down) heavy-hole excitons and right- (left-)circularly polarised photons ${ }^{3-6}$. Experimentally, their spin is directly accessible through the polarisation of the condensate emission (Suppl. 1), enabling phenomena such as non-local spin-switching ${ }^{3}$, multi-stability ${ }^{4,14}$ and the optical spin-Hall effect ${ }^{15}$. However, while electrical control of electron spins in the solid state has greatly advanced in recent years, allowing their fully integrated injection, manipulation and detection ${ }^{16}$, no such advances have been made regarding polaritons.

Here we demonstrate electrical control of the spin of polariton condensates. Spatially separating the condensate from its non-resonant pump (in a trapped geometry, Fig. 1b) diversifies the observed spin configurations, accessing circularly- to linearly-polarised states ${ }^{17}$. The condensate polarisation critically depends on several system parameters, including tiny energetic splittings in its linear polarisation. Since the latter can be tuned by electric fields, applying a bias across the microcavity provides direct control of the condensate spin. We utilise this mechanism to invert the spin of an optically-trapped condensate exhibiting bistable behaviour, thus realising an electrical spin switch. Such electro-optic switching is crucial in many communications and IT applications.

Our results are obtained from micron-long cavities at cryogenic temperatures, which contain twelve QWs and are processed into contacted mesas, allowing application of electric fields perpendicular to the QWs (Fig. 1a, methods). A spatial light modulator is used to project four laser spots onto the sample surface (Fig. 1b). The linearly-polarised, continuous-wave pump nonresonantly generates clouds of hot excitons, which in a series of relaxation steps accumulate at the bottom of the polariton dispersion and condense. Since polaritons are blue-shifted at locations of high exciton and polariton densities due to their repulsive interactions ${ }^{7}$, the laser pattern induces a square-shaped potential trap (Fig. 1b). 


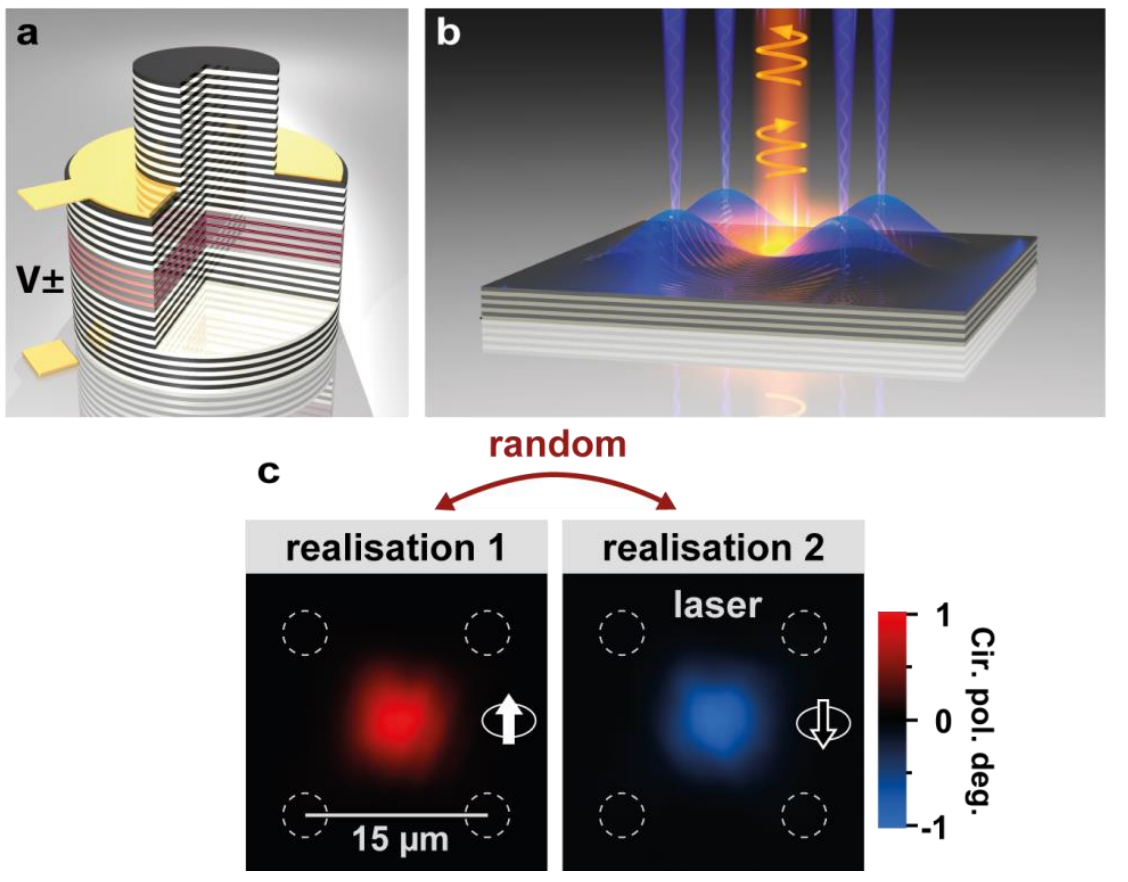

Figure 1 I Spontaneous spin-polarisation of trapped polariton condensates. a, Microcavity structure with 12 QWs sandwiched between two multilayer mirrors. Contacts are deposited on an etched annular recess and below the bottom reflector to apply fields perpendicular to the QW plane. b, Schematic of trapped polariton condensate. Four linearly polarised laser beams induce a reservoir distribution corresponding to a square-shaped potential trap. The resulting condensate is confined to the centre and shows circular polarisation. c, Polarisation-resolved spatial images of the circular component of condensate emission for two measurements under identical experimental conditions. While both measurements exhibit strong circular polarisation $\left(\left|s_{z}\right|>95 \%\right)$ which remains stable for seconds, the direction of polarisation spontaneously changes from right- (spin-up) to left-circular (spin-down) when the condensate is re-initialised. White arrows indicate condensate spin.

Such conditions favour formation of 'trapped' condensates which minimally overlap with the exciton reservoir, resulting in narrower linewidths and strongly reduced condensation thresholds ${ }^{18-20}$. Moreover, separating condensate and reservoirs leads to spontaneous breaking of the spin-symmetry for excitation powers above a spin-bifurcation threshold ${ }^{17}$. The condensate then spontaneously adopts one of two circularly-polarised bistable states under linear, nonresonant excitation, stochastically changing between right- and left-circular polarisations (spin-up and spindown) for each realisation of the experiment (Fig. 1c). Note that this spin bifucation is favoured by the anisotropy of the non-linear polariton-polariton interactions (see Eq. S3, Suppl.1) and fundamentally differs from conventional polariton bistability, where the spectral position and pump power of a resonant laser deterministically select one of the polarisation states ${ }^{4,14}$.

The influence of external electric fields on the spin of a trapped polariton condensate is explored in Fig. 2a,d. The strong circular polarisation observed at $0 \mathrm{~V}$ transforms to elliptical as the applied bias increases, until the bistable states collapse into a single linearly polarised mode around $\pm 15 \mathrm{~V}$. The orientation of the linear polarisation axes, referred to as horizontal and vertical, does not depend on the optical trap or the position on the sample surface, but is fixed along the [110] and [1필 axes of the crystal structure. The applied bias $U$ induces a small energy splitting $\hbar \epsilon_{F}$ between the horizontally and vertically polarised modes, varying linearly with the bias (Fig. 2c,e,f). Two physical mechanisms contribute to this behaviour: First, applying an electric field along the [001] axis of the crystal induces birefringence due to the Pockels effect, leading to splitting of the photonic modes linearly polarised along the [110] and [110] axes $^{21}$. Second, external fields mix heavy- and light-hole QW excitons, lifting the degeneracy of the exciton ground state ${ }^{22,23}$. Both effects split linearly-polarised polariton modes through a linear dependence on the external field with magnitudes matching the experimental data ${ }^{21,23}$. Note that the transverse-electric transverse-magnetic energy splitting observed for polaritons at larger in-plane wavevectors vanishes at normal incidence and consequently plays no role here. Moreover, TE-TM splitting does not break the spin-symmetry and can thus not account for the stochastic formation of circularly polarised condensates. 

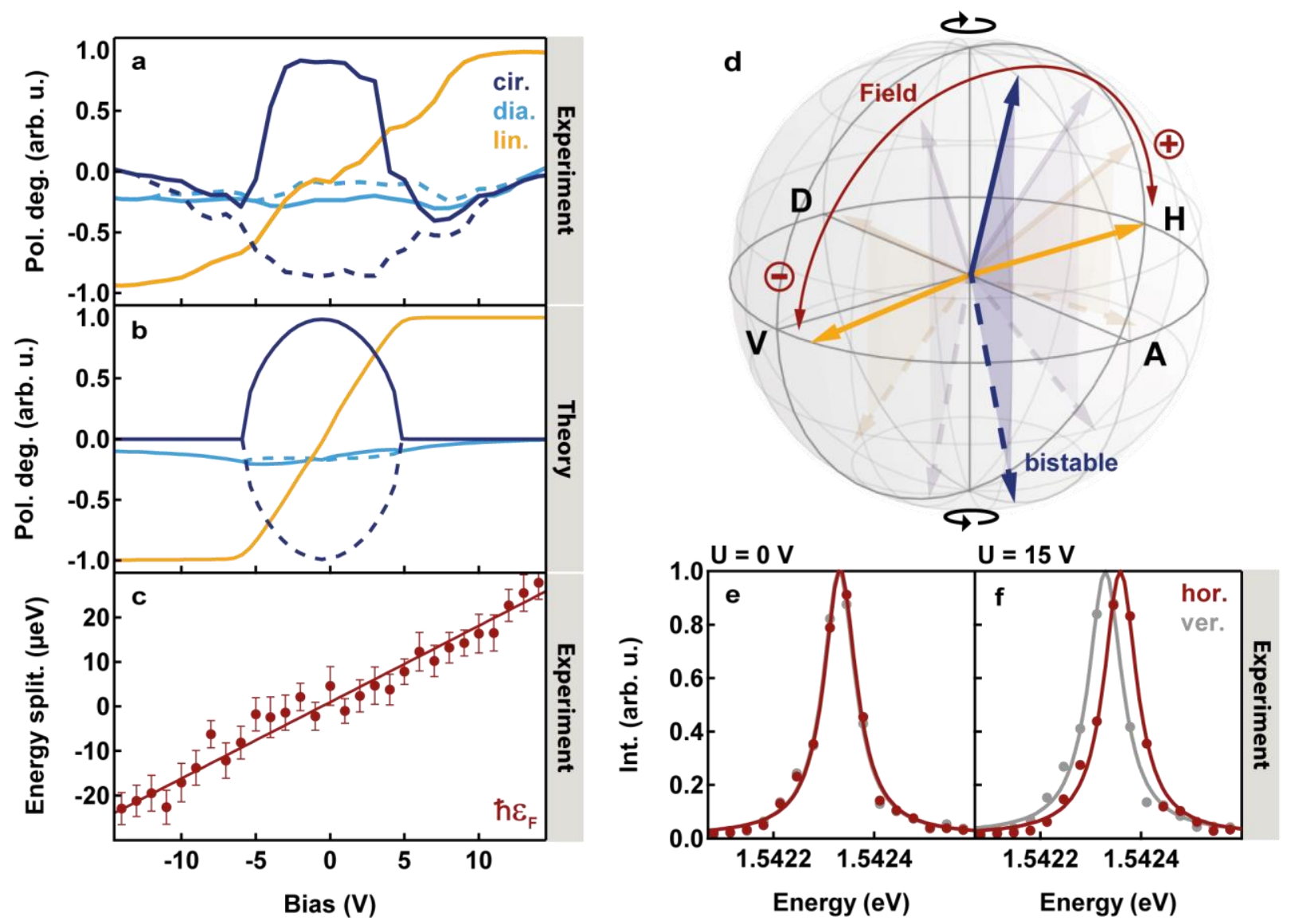

Figure 2 | Condensate polarisation under external electric fields. a, Bias dependence of polarisation components of a trapped polariton condensate. Shown is the average polarisation of bistable spin-up (solid lines) and -down (dashed lines) states obtained from 100 realisations at each bias, positive values for horizontal, diagonal and right-circular polarisations. b, Numerical simulations of the same. c, Measured energy splitting between horizontal-vertical condensate components vs applied bias $U$. Data points average 100 measurements at each bias. Linear fit gives $\hbar \epsilon_{F} \approx 0.9 \mu \mathrm{eV} \mathrm{V}^{-1} \cdot U+1.3 \mu \mathrm{eV}$. The $\hbar \epsilon_{S} \approx 1.3 \mu \mathrm{eV}$ offset at $0 \mathrm{~V}$ is attributed to the local effect of strain ${ }^{24,25}$. $\mathbf{d}$, Illustration of transition from bistable regime at $0 \mathrm{~V}$ (blue) to linear-polarised condensate at $\pm 15 \mathrm{~V}$ (yellow) on the Poincare sphere. e,f, Condensate emission spectra of horizontal and vertical components at $0 \mathrm{~V}$ and $15 \mathrm{~V}$, showing $\hbar \epsilon_{F} \approx 0 \mu \mathrm{eV}$ and $30 \mu \mathrm{eV}$, respectively.

To interpret the experimental results, we extend the model of spontaneous spin bifurcations ${ }^{17,26}$ to include the effects of an external electric field (see methods and Suppl. 1). A small energy-splitting $\hbar \epsilon$ between two linearly polarised modes with difference $-2 \gamma$ between their respective loss rates induces a dynamical instability, driving a parity-breaking bifurcation above a critical density. Physically, the observed linear anisotropies $\hbar \epsilon_{F}$ and $\hbar \epsilon_{S}$ arise due to the combined effects of electric field and strain ${ }^{21-25}$ and necessarily translate into linewidth differences $\hbar \gamma_{F, S}$ between the corresponding linear modes due to curvature of the cavity stopband (Suppl. 2). For the given microcavity structure, transfer matrix simulations predict $\gamma_{F, S} \approx$ $0.05 \epsilon_{F, S}$, below the resolution of our experiment. Numerical simulations of Eq. S4 (Suppl. 1) are based on the measured bias- dependence of $\hbar \epsilon_{S, F}$ (Fig. 2b). The good qualitative agreement with the experiment demonstrates that electrical control of the condensate spin is indeed achieved by tuning the linearly polarised condensate modes. Our model furthermore implies that the ability to bias-tune the condensate polarisation critically depends on the non-linear polariton selfinteractions, which control the transition between the bistable and the linear regime. (Suppl.1).

We now realise an electrical spin switch based on these phenomena. Slightly rotating the linear pump with respect to the crystal axes induces field-dependent birefringence (Pockels effect), giving rise to a small degree of circular polarisation in the excitation. The imbalance of the left- and right-circular pump rates results in preferential occupation of one of the two bistable condensate states when applying electric fields, leading to stable left-/right-circular polarisation above $\pm 0.3 \mathrm{~V}$, respectively (Fig. $3 a$ ). Under continuous optical excitation the condensate circular polarisation now exhibits hysteresis with applied voltage, corresponding to a bias-threshold for spin-switching. To reproduce the experimental data, a field-dependent pump-imbalance $\Delta P$ between the spin-up and -down components of the 

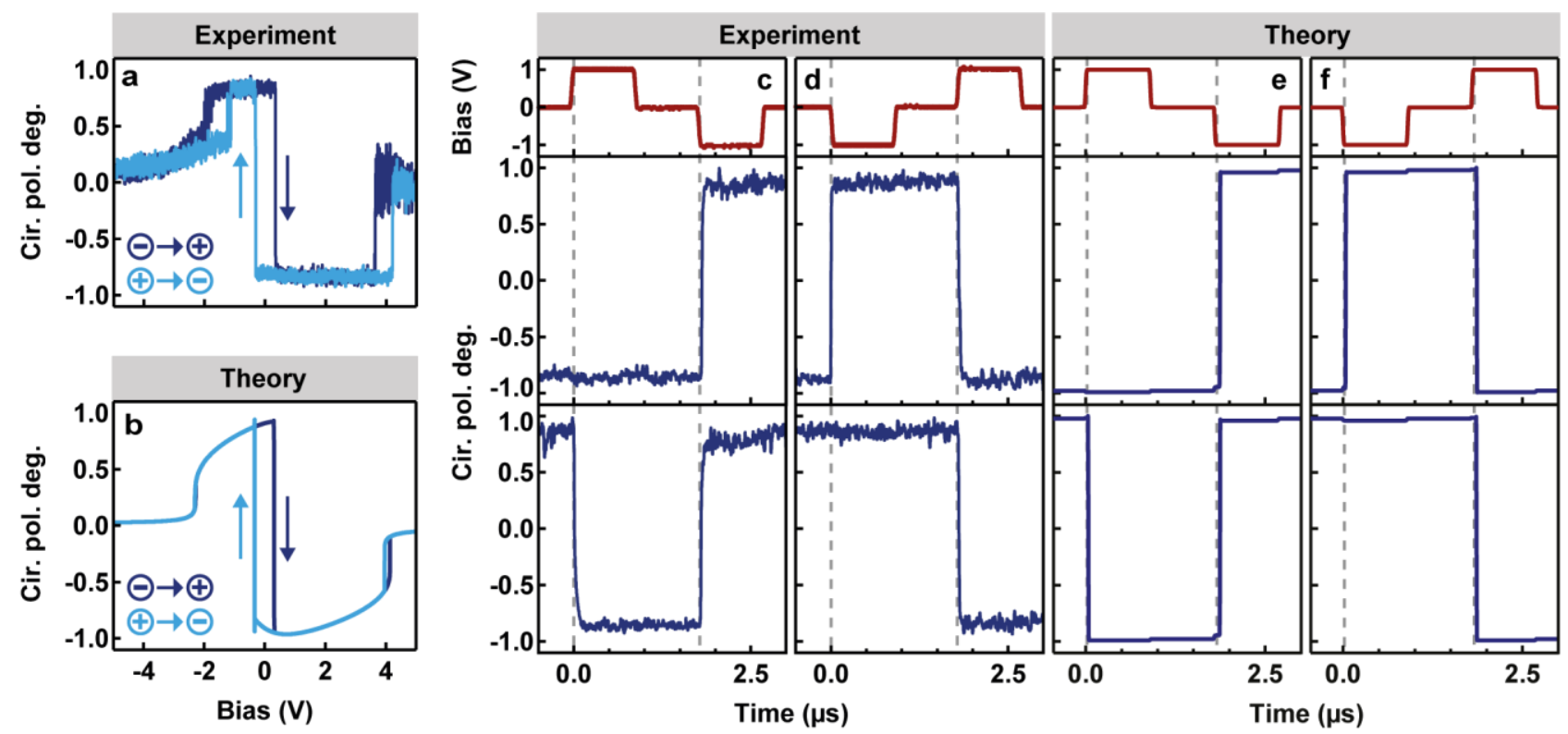

Figure 3 | Polarisation hysteresis and electrical spin-switching. a, Circular polarisation vs applied bias under continuous excitation. Bias swept from $-5 \mathrm{~V}$ to $+5 \mathrm{~V}$ (dark blue) and back (light blue) over $40 \mu$ s duration. Positive values represent right-circular polarisation. $\mathbf{b}$, Numerical simulation of a with additional bias-dependent pump imbalance $\Delta P$ in the model (Suppl. 3). c,d, Time evolution of circular polarisation (dark blue) for $\pm 1 \mathrm{~V}$ electrical pulses of $0.9 \mu$ s duration (red, top panel) acting on condensates randomly initialised in the up- (bottom panel) and down-states (middle panel). $\mathbf{e}, \mathbf{f}$, Corresponding numerical simulations based on the model of $\mathbf{b}$. Note: data obtained for different excitation conditions than Fig. 2 (see methods).
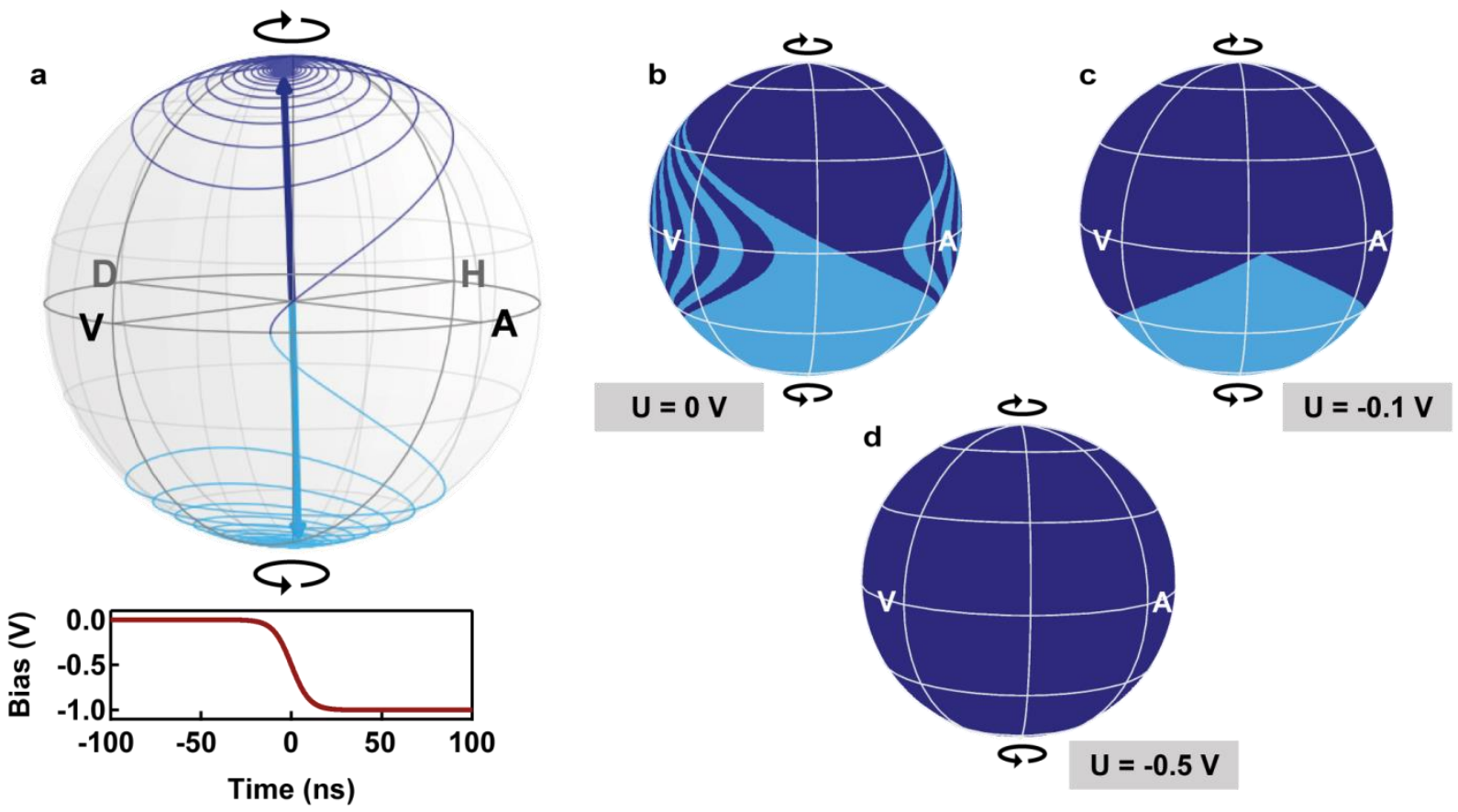

Figure 4 | Mechanism of the electrical spin-switch. a, Simulation of a switching pseudospin trajectory (from Fig. $3 f$ middle panel, for the first switching process). b, Time evolution of 500 randomly chosen polarisation states without applied electric field, depicted on the Poincare-sphere. Colours encode trajectories ending in the spin-up (dark blue) and spindown (light blue) attractors, thus approximating the respective basins of attraction. $\mathbf{c}, \mathbf{d}$, Same as $\mathbf{b}$, but for external fields of $-0.1 \mathrm{~V}$ and $-0.5 \mathrm{~V}$, respectively. Simulations demonstrate how the basin of attraction of the spin-down state is destabilised due to the applied bias (see Suppl. 3 for additional discussions). 
condensate is introduced to the model (methods and Suppl. 3), leading to a collapse of the bistable region above $\pm 0.3 \mathrm{~V}$ as well as polarisation hysteresis in the numerical simulations (Fig. 3b). The system can be widely tuned, through excitation power (bistability range), sample position (rotating the axis of straininduced splitting) and polarisation of the excitation laser (spin population imbalance).

The hysteretic nature of the system allows the implementation of electrically controlled directional spin switching (Fig. 3c,d). Electric pulses of $\pm 1 \mathrm{~V}$ are applied to condensates which are randomly initialised in the bistable region, inducing switching depending on the previous condensate polarisation. For instance, Fig. 3c illustrates that $\mathrm{a}+1 \mathrm{~V}$ pulse will only switch the spin of a condensate initialised in the right-circularly polarised state, whereas the opposite holds for a $-1 \mathrm{~V}$ pulse. Note that the condensate state persists after the voltage pulse ends, demonstrating the bistable nature of the system. Unlike conventional switches where an energy barrier controls transitions between states, switching of this nonlinear system is achieved by temporarily destabilising one of the basins of attraction due to the field-induced pump imbalance (Fig. 4). The measured switching time $t_{s} \approx 2.5 \mathrm{~ns}$ is limited by our resolution (Fig. 5) and exceeds the rise-time of the electric pulse, illustrating that the switching process is non-adiabatic.

To estimate the switching energy $E_{s}, \pm 3 \mathrm{~V}$ electrical pulses of $t_{p} \approx 4$ ns duration are applied to the sample (Fig. 5). While switching is already observed at applied fields as low as $\pm 0.3 \mathrm{~V}$ (Fig. 3a), increasing the bias significantly improves the reliability of the switching process (Suppl.4). The drawn current $I_{S} \approx 37 \mathrm{nA}$ is obtained from the device IV-curve (Suppl. 5), resulting in a switching energy of $E_{s}=V_{s} \cdot I_{s} \cdot t_{p} \approx 0.5 \mathrm{fJ}$. This estimate surpasses all state-of-the-art electronic (spin-) switches ${ }^{2,27-29}$, although more energy efficient polaritonbased all-optical solutions exist ${ }^{13,30}$. However, our estimate represents only an upper bound for the fundamental minimum switching energy in this system, since wasted energy from the photocurrent is not

\section{Materials and methods}

The sample studied is a $5 \lambda / 2 \mathrm{Al}_{0.3} \mathrm{Ga}_{0.7} \mathrm{As}$ microcavity, formed by distributed Bragg reflectors (DBRs) with 32/35 layer pairs on top/bottom. The individual layer pairs are composed of $\mathrm{Al}_{0.15} \mathrm{Ga}_{0.85} \mathrm{As} / \mathrm{AlAs}$ with thicknesses of $57.2 / 65.4 \mathrm{~nm}$, respectively. The quality factor of the microcavity exceeds $Q>16,000$. Four sets of three GaAs QWs are located at the maxima of the cavity light-field, resulting in a exciton-photon Rabi-splitting of $12 \mathrm{meV}$. The cavity mode is detuned approximately $-5 \mathrm{meV}$ relative to the exciton energy. The $200 \mu \mathrm{m}$ diameter mesas are etched into the sample and electrical contacts deposited on the $n$ - intrinsic to switching but stems from parasitic thermal escape of hot carriers. Improved sample designs could reduce the current drawn to the corresponding dark current $(\sim 1.6 \mathrm{nA})$ resulting in significantly lower switching energies (Suppl.5). The spin switch eye diagram at applied fields of $\pm 3 \mathrm{~V}$ is fully open, confirming stable operation for hundreds of cycles (Suppl. 4).

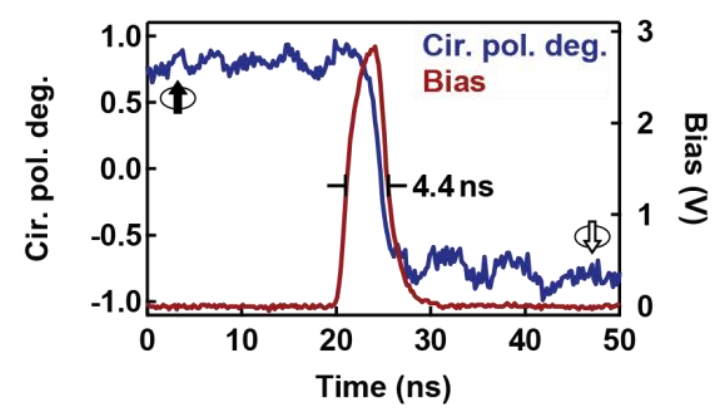

Figure 5 | Spin-switching time. Measured time evolution of the circular polarisation component (blue) under the influence of a $3 \mathrm{~V}$ bias pulse (red). The duration of the bias pulse $t_{p}$ is approximately $4.4 \mathrm{~ns}$ (FWHM). The observed condensate switching time $t_{s} \approx 2.5 \mathrm{~ns}$ is limited by the time resolution of the experimental setup.

In conclusion, we demonstrate the ability to electrically control the spin of a trapped polariton condensate and realise an electrical spin switch operating at ultra-low switching energies. The switching mechanism is highly reliable and the selected condensate states remain stable for many seconds (Suppl. 6). Our findings suggest implementation of electrically-controlled spin-optotronic devices and effects, such as polariton-spin transistors, switches and memories 23,31 , which in the case of large-bandgap materials could operate at room temperature ${ }^{32}$. Finally, from a more general point of view, our results represent the first example of electrically controlling the spin of a macroscopic quantum state, such as Bose-Einstein condensates or superconductors.

doped substrate and on an annular recess around the top of the mesa, four DBR layers above the cavity location. An applied bias $U$ provides a resulting electric field $F[\mathrm{kV} /$ $\mathrm{cm}] \approx 1.86 / \mathrm{cm} \cdot U[\mathrm{~V}]$.

Polaritons are excited non-resonantly, with a $\lambda=$ $750 \mathrm{~nm}$ horizontally-polarised, single-mode continuous wave laser at a total power of approximately $40 \mathrm{~mW}$ in four separate spots. The excitation amplitude is modulated with an acousto-optic modulator giving a rise time of 90 ns. A spatial light modulator is employed to generate patterns of laser spots on the sample surface. The resulting emission around $800 \mathrm{~nm}$ is polarisation resolved with a 
series of beam splitters, quarter- and half-waveplates and a Wollaston-prism to simultaneously measure its linear, diagonal, and circular components. The signal is recorded with a CCD for imaging and a monochromator plus CCD for spectral analysis, while photomultipliers are employed for time-resolved measurements of $7 \mathrm{~ns}$ resolution. A sourcemeasurement unit is used to apply fields across the sample and monitor the resulting currents. All measurements were performed at cryogenic temperatures $(\mathrm{T}<10 \mathrm{~K})$. For further details see Ref 33.

Simulations of Fig. 2b, Fig. 3ef and Fig. 4 are based on equations of the form (see Suppl. 1 and 3 for a detailed discussion):

$$
\begin{aligned}
i \dot{\Psi}=\quad & -\frac{i}{2} g(S) \Psi \\
& -\frac{i}{2}\left(a_{S} \sigma_{x}+b_{S} \sigma_{y}\right)\left(\gamma_{S}-i \epsilon_{S}\right) \Psi \\
& -\frac{i}{2}\left(a_{F} \sigma_{x}+b_{F} \sigma_{y}\right)\left(\gamma_{F}-i \epsilon_{F}\right) \Psi \\
& +\frac{1}{2}\left[\left(\alpha_{1}+\alpha_{2}\right) S+\left(\alpha_{1}-\alpha_{2}\right) S_{z} \sigma_{z}\right] \Psi,
\end{aligned}
$$

where $\hbar$ was set to 1 and $\Psi=\left(\psi_{+}, \psi_{-}\right)^{T} \cdot g(S)$ describes the balance between the pump-rate $P$ and the decay-rate $\Gamma$, with $g(S)=\Gamma-P+\eta S$. The term $\eta S$ accounts for gainsaturation, with the total occupation $2 S=\left|\psi_{-}\right|^{2}+\left|\psi_{+}\right|^{2}$ and the saturation factor $\eta$. In the case of an imbalanced pump, $g(S)$ takes the form $g(S)=(\Gamma-(1-\beta) P+$ $\eta S, \Gamma-(1+\beta) P+\eta S)^{T}$, with the field-induced pumpimbalance $\Delta P=2 \beta(F) P$ (see Suppl. 3 for details). The factors $\epsilon_{S, F}$ and $\gamma_{S, F}$ denote the energy splitting and

\section{References and notes:}

1. Wolf, S. A. et al. Spintronics: A Spin-Based Electronics Vision for the Future. Science 294, 14881495 (2001).

2. Reed, G. T., Mashanovich, G., Gardes, F. Y. \& Thomson, D. J. Silicon optical modulators. Nat. Photonics 4, 518-526 (2010).

3. Amo, A. et al. Exciton-polariton spin switches. Nat. Photonics 4, 361-366 (2010).

4. Paraïso, T. K., Wouters, M., Léger, Y., MorierGenoud, F. \& Deveaud-Plédran, B. Multistability of a coherent spin ensemble in a semiconductor microcavity. Nat. Mater. 9, 655-660 (2010).

5. Armitage, A. et al. Exciton polaritons in semiconductor quantum microcavities in a high magnetic field. Phys. Rev. B 55, 16395-16403 (1997).

6. Sturm, C. et al. Nonequilibrium polariton condensate in a magnetic field. Phys. Rev. B 91, 155130 (2015).

7. Wertz, E. et al. Spontaneous formation and optical manipulation of extended polariton condensates. Nat. Phys. 6, 860-864 (2010).

8. Nelsen, B. et al. Dissipationless Flow and Sharp Threshold of a Polariton Condensate with Long Lifetime. Phys. Rev. X 3, 041015 (2013).

9. Kasprzak, J. et al. Bose-Einstein condensation of exciton polaritons. Nature 443, 409-414 (2006). corresponding linewidth differences arising from sample strain and an applied electric field, respectively. The splitting axis is selected by the factors $a_{S, F}$ and $b_{S, F}$, where $a_{i}^{2}+b_{i}^{2}=1$ and $a_{i}, b_{i} \in[-1,1]$. The coefficients $\alpha_{1}$ and $\alpha_{2}$ represent the strength of same- and cross-spin polaritonpolariton interactions.

The parameters used for the numerical simulations of Fig. $2 \mathrm{~b}$ and Suppl. 1 are: $\eta=0.01 \mathrm{ps}^{-1}, \Gamma=0.2 \mathrm{ps}^{-1}, P=$ $0.205 \mathrm{ps}^{-1}, \hbar \alpha_{1}=10 \mu \mathrm{eV}$ and $\alpha_{2}=-0.5 \cdot \alpha_{1}$. The field induced energy splitting $\hbar \epsilon_{F}$ as a function of the applied bias $U$ is determined from the linear fit in Fig. 2 c as $\hbar \epsilon_{F} \approx$ $0.9 \mu \mathrm{eV} / \mathrm{V} \cdot U+1.3 \mu \mathrm{eV}$. The offset of $\hbar \epsilon_{F}$ is attributed to the effect of strain, with a maximum field-independent splitting $\hbar \epsilon_{S} \approx 1.6 \mu \mathrm{eV}$ being observed along the axes rotated by $-25^{\circ}$ relative to the horizontal/vertical ( $a_{S}=$ $-0.6)$. The corresponding linewidth differences are derived from the transfer matrix calculations presented in Suppl. 2 as $\gamma_{S, F}=0.05 \cdot \epsilon_{S, F}$. To achieve a better fit of the diagonal polarisation at large fields, the linear polarisation axes between which the splitting $\hbar \epsilon_{F}$ is induced are assumed to be rotated by $1.3^{\circ}$ with respect to the experimentally defined horizontal and vertical axes, i.e. $a_{F}=0.999$.

The data presented in Fig. 3 was obtained at a different sample position (different strain) and excitation power than that of Fig. 2, with the corresponding parameters being changed to $P=0.203 p s^{-1}$ and $a_{S}=0.6$ to match the experimental data. Additionally, a field-dependent imbalance $\Delta P$ was added to the pump-terms of the spin-up and -down components, with $\Delta P=\mp U \cdot P \cdot 0.002$. The same parameters were used for the simulations in Suppl. 3 and Suppl. 5.

10. Balili, R., Hartwell, V., Snoke, D., Pfeiffer, L. \& West, K. Bose-Einstein Condensation of Microcavity Polaritons in a Trap. Science 316, 1007-1010 (2007).

11. Sanvitto, D. et al. Persistent currents and quantized vortices in a polariton superfluid. Nat. Phys. 6, 527533 (2010).

12. Gao, T. et al. Polariton condensate transistor switch. Phys. Rev. B 85, 235102 (2012).

13. Ballarini, D. et al. All-optical polariton transistor. Nat. Commun. 4, 1778 (2013).

14. Cerna, R. et al. Ultrafast tristable spin memory of a coherent polariton gas. Nat. Commun. 4, (2013).

15. Leyder, C. et al. Observation of the optical spin Hall effect. Nat. Phys. 3, 628-631 (2007).

16. Awschalom, D. D. \& Flatté, M. E. Challenges for semiconductor spintronics. Nat. Phys. 3, 153-159 (2007).

17. Ohadi, H. et al. Spontaneous Spin Bifurcations and Ferromagnetic Phase Transitions in a Spinor ExcitonPolariton Condensate. Phys. Rev. X 5, 031002 (2015).

18. Cristofolini, P. et al. Optical Superfluid Phase Transitions and Trapping of Polariton Condensates. Phys. Rev. Lett. 110, 186403 (2013).

19. Dreismann, A. et al. Coupled counterrotating polariton condensates in optically defined annular 
potentials. Proc. Natl. Acad. Sci. 111, 8770-8775 (2014).

20. Askitopoulos, A. et al. Polariton condensation in an optically induced two-dimensional potential. Phys. Rev. B 88, 041308 (2013).

21. Park, M. S. et al. Polarization control of verticalcavity surface-emitting lasers by electro-optic birefringence. Appl. Phys. Lett. 76, 813-815 (2000).

22. Aleiner, I. L. \& Ivchenko, E. L. Anisotropic exchange splitting in type-II GaAs/AIAs superlattices. J. Exp. Theor. Phys. 55, 692 (1992).

23. Malpuech, G., Glazov, M. M., Shelykh, I. A., Bigenwald, P. \& Kavokin, K. V. Electronic control of the polarization of light emitted by polariton lasers. Appl. Phys. Lett. 88, 111118 (2006).

24. Kłoptowski, L. et al. Optical anisotropy and pinning of the linear polarization of light in semiconductor microcavities. Solid State Commun. 139, 511-515 (2006).

25. Balili, R. et al. Huge splitting of polariton states in microcavities under stress. Phys. Rev. B 81, 125311 (2010).

26. Aleiner, I. L., Altshuler, B. L. \& Rubo, Y. G. Radiative coupling and weak lasing of exciton-polariton condensates. Phys. Rev. B 85, 121301 (2012).

27. Wada, O. Femtosecond all-optical devices for ultrafast communication and signal processing. New J. Phys. 6, 183 (2004).

28. Nozaki, K. et al. Sub-femtojoule all-optical switching using a photonic-crystal nanocavity. Nat. Photonics 4, 477-483 (2010).

29. Fiori, G. et al. Electronics based on two-dimensional materials. Nat. Nanotechnol. 9, 768-779 (2014).

30. Marsault, F. et al. Realization of an all optical exciton-polariton router. Appl. Phys. Lett. 107, 201115 (2015).

31. Shelykh, I. A., Johne, R., Solnyshkov, D. D. \& Malpuech, G. Optically and electrically controlled polariton spin transistor. Phys. Rev. B 82, 153303 (2010).

32. Christmann, G., Butté, R., Feltin, E., Carlin, J.-F. \& Grandjean, N. Room temperature polariton lasing in a GaN/AIGaN multiple quantum well microcavity. Appl. Phys. Lett. 93, 051102 (2008).

33. Tsotsis, P. et al. Tuning the Energy of a Polariton Condensate via Bias-Controlled Rabi Splitting. Phys. Rev. Appl. 2, 014002 (2014).

\section{Acknowledgements:}

We acknowledge grants EPSRC EP/L027151/1, EU INDEX 289968, ERC LINASS 320503, Spanish MEC (MAT200801555), Mexican CONACYT 251808 and EU FP7-REGPOT2013-1 grant agreement 316165 II and Fundación La Caixa. We thank Bart de Nijs for discussions. 